\title{
In Vivo Quantum Dot Labeling of Embryonic Neural Precursor Cells
}

\author{
Jonathan R. Slotkin1,4*, Lina Chakrabarti2*, Hai Ning Dai1, G. Ian Gallicano3, \\ Barbara S. Bregman1, Joshua G. Corbin1 and Tarik F. Haydar2
}

Departments of 1Neuroscience and 3Cell Biology, Georgetown University Medical School, Washington, DC, USA; 2Center for Neuroscience Research, Children's Research Institute, CNMC, Washington, D.C. 20010; 4Department of Neurosurgery, The Brigham and Women's Hospital, Children's Hospital Boston, Harvard Medical School

Quantum dots (QD, fluorescent semiconductor nanocrystals) are a recently developed class of inorganic fluorophore, which hold great promise for biomedical research and clinical applications. QD have significant advantages compared to conventional fluorophores including: increased quantum efficiency, broader absorption spectra, narrower emission spectra, and resistance to photo bleaching. Several studies have demonstrated that cells loaded with QD in vitro can be detected when injected in vivo. However, neither direct in vivo labeling of cells with QD nor the use of QD labeling in the mammalian nervous system have been achieved. $\mathrm{Cd} / \mathrm{Se}$ core, $\mathrm{Zn} / \mathrm{S}$ shell QD in a biocompatible coating were used in these studies (Evident Technologies). QD were microinjected in vivo into the brains of embryonic day 14 mice in one of three ways: 1) High-resolution ultrasound (HRU) guided injection of QD into the ventral telencephalon, 2) HRU-guided injection into the lateral ventricle, or 3) injection into the lateral ventricle followed by co-electroporation with plasmid DNA encoding GFP. QD were prepared with PEG, transferrin, and carboxyl coatings. Embryos were harvested 2-4 days after QD injection. Confocal and multiphoton microscopy demonstrated that a population of neural precursor cells were successfully labeled with QD and could be readily detected. All methods tested for injection of quantum dots and all quantum dot variants were successful for in vivo QD labeling of individual neural precursor cells. When different color quantum dots were co-transplanted, they could be detected simultaneously using a single infrared excitation wavelength followed by spectral unmixing. We demonstrate for the first time that QD can be used for direct in vivo cellular labeling. This novel approach will facilitate ongoing studies on cell division, fate determination, and cellular migration. Support Contributed By: NIH NS27054,NICHD HD007459,NS051852,AANS/CNS

Figure1: The composition and spectral characteristics of quantum dots.(A) The QDs have CdSe core/ZnS shell covered by a phospholipid coat to which ligands, amine and carboxy groups can be added to functionalize the QDs. (B, C) N2a cells were loaded with an equimolar mix of $490 \mathrm{~nm}, 520 \mathrm{~nm}, 580 \mathrm{~nm}$ and 620nm QDs in vitro using Lipofectamine (LF) 2000. 24 hours later the cells were imaged using multiphoton excitation at $800 \mathrm{~nm}$ and detected with Zeiss Meta detector. The emission fingerprints of the four QDs are graphed in (B) and an unmixed lambda stack image is shown in (C). (D, E) N2a cells were simultaneously loaded with 620nm QDs and transfected with EGFP-F using LF2000. The region of interest (dotted area) in (D) was scanned 120 times using a 488nm argon laser to excite EGFP-F and a $633 \mathrm{~nm}$ HeNe laser for the QD excitation. Traces of fluorescence intensity vs. time in (E) demonstrate that the QD emission increased slightly during repetitive illumination while EGFP fluorescence faded.

Figure 2: Cytotoxicity assays. (A) Lactic acid dehydrogenase (LDH) activity was measured in the culture media of $620 \mathrm{~nm}$ QD loaded N2a cells at different time points in vitro. Compared to positive (freeze-thaw, [F/T]) and negative (media alone or LF2000 [LF] treated) control cultures, 
QD loading does not show any appreciable change in LDH release, regardless of the concentration of QD used
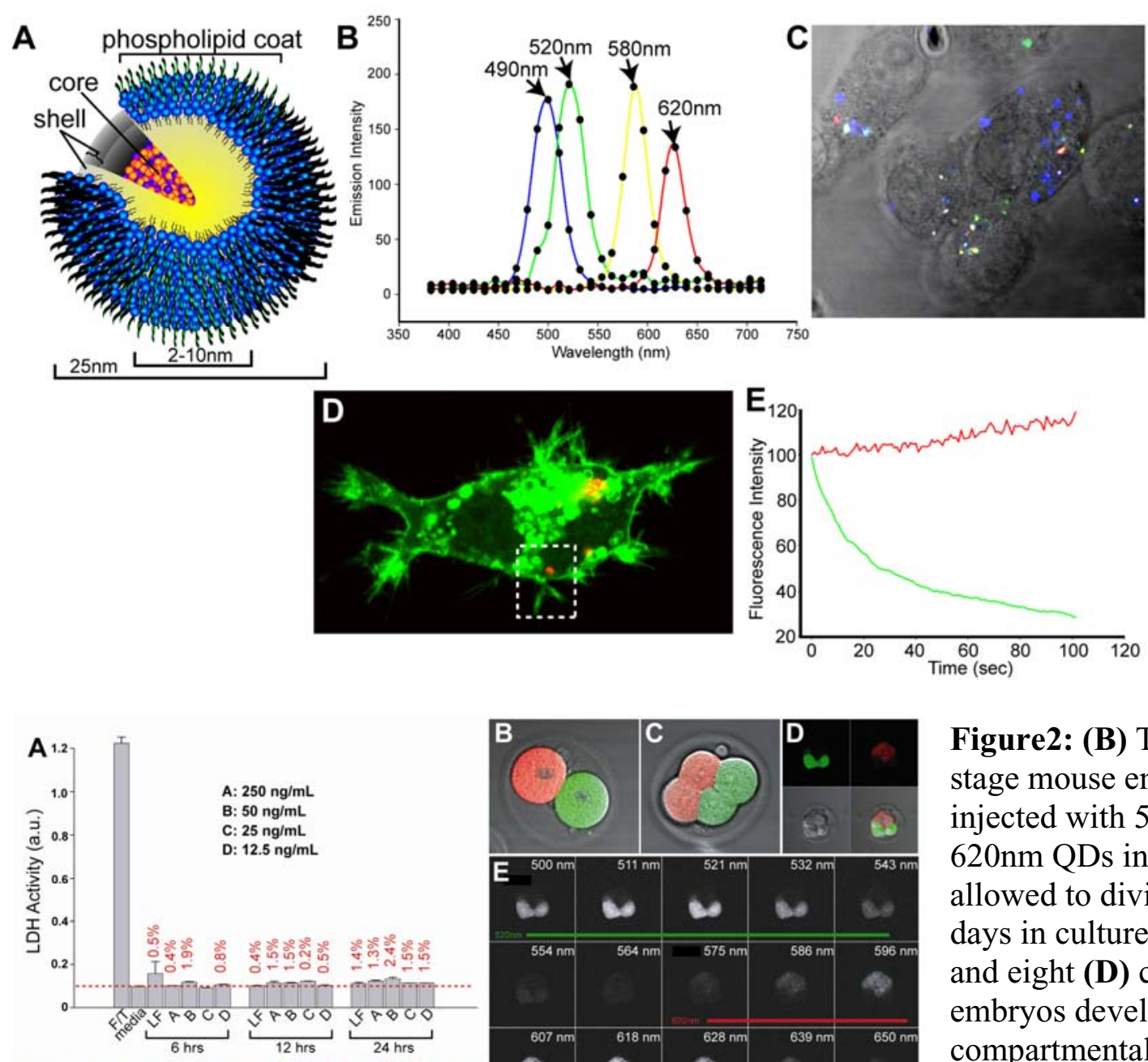

Figure2: (B) Two cell stage mouse embryos injected with $520 \mathrm{~nm}$ and

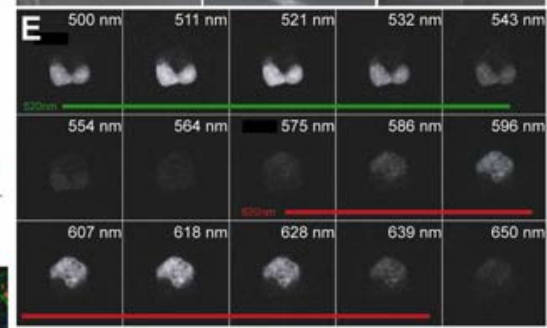
$620 \mathrm{~nm}$ QDs in vitro were allowed to divide for 1-2 days in culture. Four (C) and eight (D) cell stage embryos developed with compartmentalized
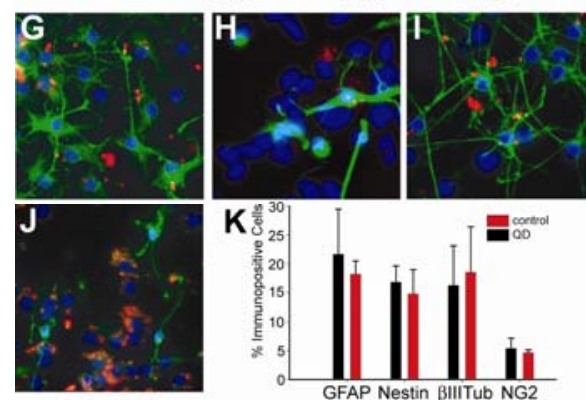

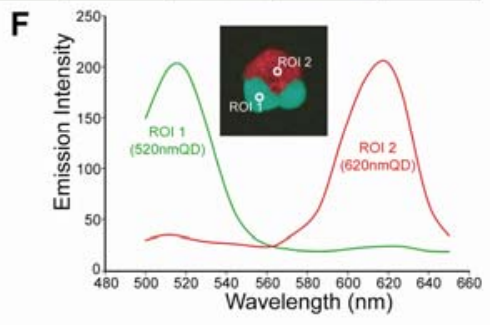

segregation of QD into daughter cells during development demonstrating there was no mixing of QD during cell division. (E) Lambda stack image illustrates the spectral characteristics of each QD.

520nm and 620nm QD. (G-K) To track the differentiation of QD loaded cells in culture, neurospheres cultured from embryonic day (E) 14.5 ventral telencephalon were dissociated and the resulting cells were labeled with 620nm QD using LF2000 for one day in proliferation medium. After 7 days of subsequent culture in differentiation medium, the cells were immunostained for nestin (G), $\beta$ IIII-tubulin (H), GFAP (I) and NG2 (J). (K) No significant difference in the percentage of immunopositive cells for any antigen was found after neural stem cell differentiation. 